\section{Better deader}

\section{By Tim Fulmer, Senior Writer}

Traditional strategies for designing live attenuated vaccines have been empirical, involving repeated passage of viral and bacterial strains through nonhuman cell lines to select for mutations that reduce pathogenicity, an approach that rarely provides insight into the molecular basis for attenuation. Thus, techniques for generating live attenuated strains have been hit or miss.

Two recent papers published in Science and the Proceedings of the National Academy of Sciences illustrate methods that could potentially be applied to designing live attenuated vaccines for a wide range of pathogens.

\section{A less fit virus}

In the Science paper, Eckard Wimmer and colleagues at Stony Brook University describe a way to design live attenuated poliovirus that could avoid the costly and time-consuming traditional approach of attenuating strains via multiple passage through cultured monkey kidney cells. Wimmer is a professor of molecular genetics and microbiology at Stony Brook.

The technique takes advantage of synonymous codon bias, a fundamental redundancy built into the coding regions of genes in all organisms. Within every gene, multiple codons can encode the same amino acid-a biological adaptation that ensures the correct rate and accuracy of protein synthesis. Although some of these codons may occur more or less frequently than others, they are all considered synonymous because replacing one codon with the other results in the incorporation of an identical amino acid into the protein.

In previous work with poliovirus, the Stony Brook group hypothesized that engineering rare or suboptimally biased codons into a viral genome could reduce replication fitness and result in a live attenuated viral strain.

Indeed, although the resulting viral strains encoded a full complement of wild-type proteins, the researchers found the strains also had lower rates of protein synthesis and translation. Most importantly, the strains showed attenuated infectivity in cultured cells and in mouse spinal cords compared with strains that were not suboptimally biased. ${ }^{1}$

In the Science paper, the same researchers extended their strategy to include pairs of adjacent codons in the poliovirus genome rather than single codons. Frequencies of adjacent codon pairs are also biased-a phenomenon called codon pair bias. ${ }^{2}$

The group engineered hundreds of suboptimally biased codon pairs into the viral capsid gene. The result was a series of poliovirus strains that all encoded wild-type proteins but had varying degrees of viral attenuation, as judged by lower growth kinetics and infectivity compared with what was seen in virus that was not deoptimized. ${ }^{3}$

Two of the attenuated strains were capable of eliciting sufficient levels of antibodies to protect mice from death or paralysis following challenge by a lethal intramuscular dose of wild-type poliovirus.

The authors concluded that their strategy, dubbed synthetic attenuated virus engineering (SAVE), "could play a role in creating new vaccines for various types of viruses. By deoptimizing codon pair bias, one could systematically attenuate a virus to variable but controllable and predictable extents."

Steffen Mueller, an author on the paper and a researcher in Wimmer's lab, said the technique may be especially useful for creating attenuated strains of newly emerging viruses in which biological details such as life cycle are unknown.

"All we need to have is the virus's genome sequence so that we can recode it synthetically and a reverse-genetics system to convert this synthetic sequence back into a virus," he said. "Such systems are available for many of the major virus types."

Mueller also said SAVE is quicker than current attenuation techniques. "With a little more practice, it may be possible in the near future to design and produce a vaccine candidate against a newly emerging virus threat within a matter of weeks," he said.

Traditional techniques of attenuation rely on spontaneous mutations occurring in the viral genome. This involves extensive passage of a pathogen through tissue culture under suboptimal conditions and can take months or even years. Moreover, there is no guarantee the process will produce a strain that is genetically stable or properly attenuated in humans, said Sims Kochi, director of bacterial vaccines at Avant Immunotherapeutics Inc.

"The Science paper addresses these issues through a rational attenuation process that introduces dozens or hundreds of codon alterations to reduce the possibility that the virus will randomly revert to a more virulent phenotype, while ensuring expression of wild-type antigens for immunogenicity and protection against challenge," noted Kochi.

\section{Attenuating the virus}

Researchers polled by SciBX want to see proof of concept in other viruses.

"It would now be useful to determine how many of these hundreds of codon alterations are in fact necessary for virus attenuation," said George Kemble, VP of R\&D and general manager of vaccines at MedImmune Inc., a subsidiary of AstraZeneca plc. "By finding out what minimal changes to the attenuated strain are sufficient to revert it back to a wild-type level of pathogenicity, the researchers might be able to dissect the molecular mechanism underlying attenuation in their model." 
Kemble also thinks that "looking at effects on attenuation caused by codon changes in functional rather than structural genes might also provide insights into the mechanism."

In the Science paper, the researchers introduced suboptimal codons only into the $\mathrm{P} 1$ region of the poliovirus genome, which encodes a capsid precursor protein that is processed into several structural proteins. However, the poliovirus genome consists of genes that encode multiple structural and functional proteins. Introducing suboptimal codons into the RNA polymerase gene, for example, could have interesting effects on attenuation, Kemble said.

Michael Watson, EVP of R\&D at Acambis plc, emphasized that "poliovirus is pathogenic to only a small minority of people. Thus, even if the strategy works in this context to generate an attenuated strain, there remains the question of whether it can be used to create safely attenuated vaccines against much more pathogenic viruses."

Acambis' ChimeriVAX-JE Japanese encephalitis (JE) vaccine met the primary endpoint of immunogenicity in a 2006 Phase III trial. The vaccine's chimeric virus consists of structural genes derived from an attenuated JE strain inserted into a yellow fever virus vector that drives replication. "The resulting vaccine could thus be described as a cross between a standard subunit vaccine and a live attenuated vaccine," said Watson.

According to Kemble, additional work is necessary to demonstrate the general applicability of the codon deoptimization strategy. "In particular, I would suggest looking at viruses with larger genomes and more complex life cycles than poliovirus, such as herpes virus," he said.

Kochi agreed with the need to look at other virus types. "Viral species with a stable genome may be well suited for this method-for example, hepatitis B virus." However, applicability should also be tested in viruses with high mutation rates, such as HIV, or viruses that undergo significant antigenic drift, as occurs with the influenza virus hemagglutinin A antigen, he said.

Watson told SciBX that safety could be a bigger concern with more virulent viruses.

"The attenuated poliovirus strain is still expressing wild-type proteins and therefore presumably targeting the same tissues as the nonattenuated virus. The strategy therefore relies on attenuation to occur by reducing the fitness or replication rate of the virus within those tissues," he said. "However, for highly pathogenic viruses, simply engineering suboptimal codons into the genome may not be enough to reduce pathogenicity and achieve safe levels of attenuation."

Watson also said it needs to be shown that SAVE can "generate an attenuated vaccine for safe use in immunocompromised individuals. Consequently, next steps ought to include evaluating the strategy in animal models of universally pathogenic diseases such as yellow fever and also in a severely immunocompromised animal model of poliovirus that could have much higher levels of viremia than the mice in the paper."

Mueller told SciBX that having shown proof of principle in poliovirus, the researchers are testing the deoptimization strategy on an undisclosed, "medically important" virus.

\section{Maintaining immunogenicity}

Researchers also wanted to see whether SAVE could generate a properly immunogenic vaccine.

Kochi told SciBX that one shortcoming of the currently used polio vaccine is that it does not provide adequate mucosal immunity, allowing poliovirus to replicate in the gut of immunized individuals and thereby producing poliovirus carriers, who could be a source of transmission. The question is whether a vaccine produced by codon deoptimization will have the same deficiency or will in fact be able to stimulate mucosal immunity, he said.

The design of FluMist, a live attenuated intranasal influenza vaccine marketed by MedImmune, provides a good example of why this can be important. Serial passage of wild-type influenza virus in cell culture at successively lower temperatures resulted in mutated strains that replicate only at cooler temperatures as occur in the nasal passages, but not at higher body temperatures.

In this way, the vaccine provides strong mucosal immunity, without risk of systemic infection, said Eun-Chung Park, program officer in the Division of Microbiology and Infectious Disease at the NIH's National Institute of Allergy and Infectious Disease.

Traditional strategies have relied on adaptive mutations to create attenuated strains that replicate in the mucosa but not in other tissues.

\section{Bacterial containment}

The second paper, published in PNAS, illustrates the strategy of using a live attenuated bacterium to deliver antigens derived from a different bacterial pathogen.

In previous work, Roy Curtiss and colleagues engineered live attenuated Salmonella enterica serovar Typhimurium with stably maintained plasmids that overexpressed the pneumococcal surface protein A (PspA) from Streptococcus pneumoniae. ${ }^{4,5}$

Curtiss is a professor in the School of Life Science and director of the Center for Infectious Diseases and Vaccinology at Arizona State University.

In the PNAS paper, his group further modified the recombinant attenuated Salmonella vaccine to ensure that the bacterium underwent lysis and released Streptococcal antigen only within host lymphoid tissue. Thus, they created a system of biological containment that could potentially prevent viral shedding of the vaccine strain into the environment without negatively affecting immunogenicity.

This was accomplished by designing an activator-promoter system that ensured Salmonella growth only in the presence of arabinose. In the absence of the sugar, as occurs in host lymphoid tissue, the bacterium would die and undergo lysis, releasing antigen cargo into the extracellular space and eliciting a host immune response.

In mice, oral delivery of the Salmonella-based vaccine elicited significantly higher levels of serum IgG and vaginal IgA against Streptococcal PspA than did mock control vector $(p<0.05)$. Serum IgG and vaginal IgA were also induced against Salmonella outer membrane proteins. ${ }^{6}$

Jan ter Meulen, head of vaccine basic research at Merck \& Co. Inc.'s Merck Research Laboratories unit, pointed out that use of live bacterial vaccines to deliver heterologous antigens have previously produced only 
weak immune responses in human trials. One of the potential pitfalls with the strategy is that Salmonella expressing heterologous antigens may be metabolically compromised and genetically unstable, ter Meulen said.

Merck markets Pneumovax 23, a vaccine to prevent Streptococcal pneumoniae infection.

Curtiss told SciBX that a dose-escalating Phase I trial, due to start in a few months, will evaluate vaccines consisting of one of three different attenuated S. enterica strains that carry plasmids expressing an antigenic domain of Streptococcal PspA. Efficacy will be measured by levels of serum and mucosal antibodies against PspA and Salmonella surface antigens.

"In the much longer term, we plan to design second-generation attenuated Salmonella-based vaccines that will express up to six different Streptococcal proteins and thus potentially elicit an even more robust immune response than the vaccine that will soon be in the clinic," he said.

Curtiss' lab is also investigating the potential use of the attenuated Salmonella system as a delivery vehicle for DNA vaccines.

In 2000, Avant acquired Megan Health Inc., a company focused on bacterial vaccine development that was founded by Curtiss. At the time, Avant said the acquisition included technology for designing vaccines against Campylobacter and Escherichia coli.
Avant has a bivalent live attenuated vaccine in preclinical development that combines a strain of Vibrio cholerae with a strain of enterotoxigenic E. coli, the causative agent of traveler's diarrhea.

\section{REFERENCES}

1. Mueller, S. et al. J. Virol. 80, 9687-9696 (2006)

2. Buchan, J. et al. Nucleic Acids Res. 34, 1015-1027 (2006)

3. Coleman, J. et al. Science; published online June 27, 2008; doi:10.1126/science. 1155761

Contact: Eckard Wimmer, Stony Brook University, Stony Brook, N.Y.

e-mail: ewimmer@ms.sunysb.edu

4. Nayak, A. et al. Infect. Immun. 66, 3744-3751 (1998)

5. Kang, H. et al. Infect. Immun. 70, 1739-1749 (2002)

6. Kong, W. et al. Proc. Natl. Acad. Sci. USA; published online July 8, 2008; doi:10.1073/pnas.0803801105

Contact: Roy Curtiss, Arizona State University, Tempe, Ariz. e-mail: rcurtiss@asu.edu

COMPANIES AND INSTITUTIONS MENTIONED

Acambis plc (LSE:ACM), Cambridge, U.K.

Arizona State University, Tempe, Ariz.

AstraZeneca plc (LSE:AZN; NYSE:AZN), London, U.K.

Avant Immunotherapeutics Inc. (NASDAQ:AVAN), Needham, Mass.

MedImmune Inc., Gaithersburg, Md.

Merck \& Co. Inc. (NYSE:MRK), Whitehouse Station, N.J.

National Institute of Allergy and Infectious Disease, Bethesda, Md.

National Institutes of Health, Bethesda, Md.

Stony Brook University, Stony Brook, N.Y. 